\title{
Construire une cartographie des expériences vécues. Récits migratoires et cartes d'itinéraires d'exilés syriens en Jordanie
}

David Lagarde

\section{OpenEdition}

Édition électronique

URL : http://journals.openedition.org/mappemonde/4568

DOI : $10.4000 /$ mappemonde.4568

ISSN : 1769-7298

Éditeur

UMR ESPACE

Référence électronique

David Lagarde, «Construire une cartographie des expériences vécues. Récits migratoires et cartes d'itinéraires d'exilés syriens en Jordanie », Mappemonde [En ligne], 129 | 2020, mis en ligne le 10 novembre 2020, consulté le 26 novembre 2020. URL : http://journals.openedition.org/mappemonde/ 4568 ; DOI : https://doi.org/10.4000/mappemonde.4568

Ce document a été généré automatiquement le 26 novembre 2020.

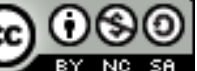

La revue Mappemonde est mise à disposition selon les termes de la Licence Creative Commons Attribution - Pas d'Utilisation Commerciale - Partage dans les Mêmes Conditions 4.0 International. 


\title{
Construire une cartographie des expériences vécues. Récits migratoires et cartes d'itinéraires d'exilés syriens en Jordanie
}

\author{
David Lagarde
}

\author{
Référence de la thèse \\ LAGARDE D. (2018). Sur les routes de l'exil syrien : récits de vie et parcours migratoires des \\ réfugiés de Deir Mqaren. Géographie. Université Toulouse le Mirail-Toulouse II.
}

1 Avant le début de l'insurrection, les ressortissants syriens pouvaient se rendre librement en Jordanie, à la simple condition d'être munis d'un passeport. Pendant plusieurs décennies, cette politique de libre circulation constitua la clé de voute d'une activité marchande génératrice de l'essentiel des revenus des habitants de Deir Mqaren ${ }^{1}$. Les hommes de ce village avaient en effet l'habitude de passer régulièrement la frontière pour se rendre dans les villes jordaniennes, où ils revendaient de petites denrées alimentaires qu'ils se procuraient en Syrie. Au cours de l'année 2012, face à l'afflux constant de personnes cherchant à fuir les violences et la crise économique engendrées par la guerre, les autorités jordaniennes ont commencé à introduire des directives visant à limiter et à mieux contrôler les mobilités des candidats à l'exil. À l'instar des combats en Syrie, ces mesures ont directement impacté les conditions de circulation des Syriennes et des Syriens dans et à proximité de l'espace frontalier syrojordanien.

2 Afin de donner à voir les effets de cette situation sur les mobilités des colporteurs de Deir Mqaren et de leurs familles, partis vivre en Jordanie en raison du conflit, j'ai construit (Lagarde, 2018) une "cartographie d'itinéraires" (Besse, 2010) destinée à « rendre compte du mouvement dans ses dimensions qualitatives et sensibles » (Bacon et al., 2016), en me basant sur les récits que ces femmes et ces hommes produisent à propos de leurs voyages entre les deux pays. 
Collecter des informations portant sur des aspects intimes et parfois douloureux de la vie des enquêtés nécessite d'établir avec eux de véritables relations de confiance. En 2014, j'ai commencé par aller à la rencontre de plusieurs familles de Deir Mqaren réfugiées en Jordanie. Cela m'a permis de passer du temps avec elles, de faire leur connaissance et de les interroger dans le cadre d'entretiens semi-directifs. Cette phase de découverte mutuelle m'a offert l'opportunité d'être clairement identifié auprès de la plupart des membres de cette communauté villageoise en exil, ce qui a largement facilité ma collecte de données lors de mon retour sur le terrain l'année suivante. En effet, durant cette seconde phase d'entretiens, j'ai pu adresser aux personnes interrogées des questions précises sur les dimensions spatiales, relationnelles et temporelles de leurs périples. En m'inspirant de la méthode des "récits de pratiques » (Grossetti, 2011), j'ai réussi à reconstituer des chaînes d'acteurs favorisant la connexion entre des localités distantes (Tastevin et Pliez, 2015) qui, mises bout à bout, forment l'ossature de leurs itinéraires. Autrement dit, lorsque les enquêtés faisaient référence à une nouvelle étape de leur voyage, je m'efforçais de leur faire préciser le moyen de transport emprunté et le temps nécessaire pour s'y rendre, ainsi que les individus mobilisés - localement ou à distance - afin de faciliter leur déplacement et leur accès aux ressources afférentes - moyen de transport, argent, informations, logement.

Dans l'optique de réussir le passage entre le récit et la carte, j'ai établi une typologie de l'ensemble des lieux et des chemins mentionnés, auxquels j'ai associé des symboles et des tracés de couleurs différentes, définies à la fois par les émotions perceptibles dans la voix et les mots de mes interlocuteurs au moment de l'entretien et leur signification dans les représentations collectives. Les camps de réfugiés apparaissent ainsi en rouge, afin d'évoquer la contrainte que représente le passage par ces lieux. À l'inverse, les logements d'amis ou de membres de la famille dans lesquels les enquêtés ont fait étape sont symbolisés par un point bleu, une couleur qui évoque le calme. J'ai aussi cherché à mettre en avant la diversité des acteurs ayant influencé leur inscription sur les différents segments de route empruntés. Figurent ainsi les déplacements " volontaires ", en bleu, ceux encadrés par des organismes chargés de canaliser les flux, en vert, ou encore ceux déterminés par des passeurs, en jaune ou en rouge, selon qu'ils ont été effectués à bord d'un véhicule ou à pied. Enfin, dans le but de suggérer le caractère approximatif d'une donnée portant sur des déplacements au tracé parfois imprécis, j'ai eu recours à des « esquisses » (Rekacewicz et Tratnjek, 2016) de lignes afin de représenter les trajets effectués dans le désert ou à travers champs.

5 J'ai également dessiné des frises spatio-temporelles, afin d'évoquer la chronologie de ces parcours. Pour établir un lien direct entre l'espace de la carte et le temps de la frise, j'ai réutilisé les symboles représentant les étapes du déplacement sous la forme de cercles proportionnels. Viennent enfin les informations d'ordre relationnel, qui permettent de montrer comment les réseaux personnels des enquêtés et de leurs proches contribuent à connecter les différentes étapes de leurs voyages. Pour chaque séquence clé du parcours, des diagrammes spécifiant la localisation des acteurs, le sens de leur mise en relation et la nature des ressources auxquelles ils facilitent l'accès viennent ainsi souligner l'importance des réseaux sociaux sur la structuration des itinéraires des réfugiés.

6 En offrant la possibilité de comparer des parcours effectués à des moments distincts, ces cartes permettent de visualiser en un seul «coup d'œil» les conséquences du durcissement progressif des politiques migratoires jordaniennes sur les différentes 
dimensions des mobilités des réfugiés. En effet, si l'on confronte les voyages de Hamza (figure 1), de sa femme Alaa (figure 2) et de sa sœur Yasmin (figure 3), effectués entre le début de l'année 2013 et la fin de l'année 2014, on constate un "glissement" considérable des routes de l'exil vers des espaces désertiques, plus difficiles d'accès et isolés du regard. La durée des déplacements et le nombre d'étapes - notamment dans des lieux de contrôle et d'enfermement tels que des camps de réfugiés, des centres de transit ou d'enregistrement - deviennent plus importants, tandis que le temps passé dans chacune d'elles augmente de façon concomitante. Sur le plan des relations mobilisées pour se déplacer sur le territoire syrien, passer la frontière jordanienne ou encore quitter un camp de réfugiés, le nombre d'acteurs sollicités augmente lui aussi nettement, forçant les exilés à se détourner de leurs seules relations personnelles afin d'accéder aux ressources facilitant leur mobilité (figure 4). Face à un contexte migratoire aussi coercitif, les candidats à l'exil doivent s'en remettre aux services de passeurs familiers du désert, mais avec lesquels ils n'entretiennent pas de liens directs ni aucune relation de confiance.

Figure 1. Itinéraire de Hamza
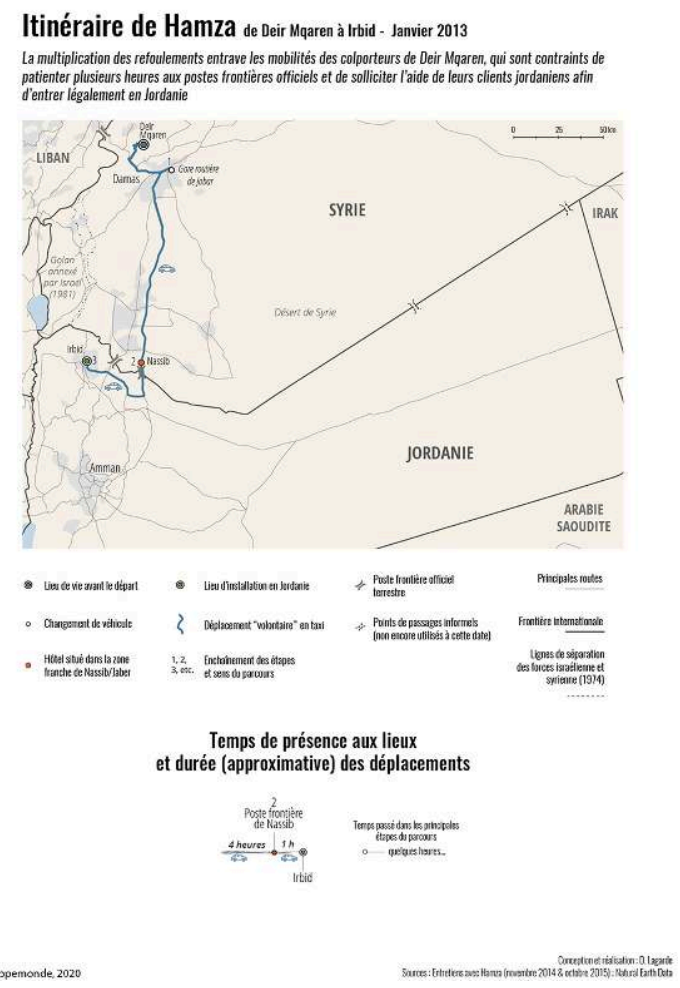
Figure 2. Itinéraire de Alaa

Itinéraire d'Alaa de Deir Mqaren à Irbid - Juin 2013 La fermeture des frontiereses officielles favorise l'émergence de nowveaux acteurs et de nouvelles routes de lexode
duc côté occidental de la zone frontafiere syro-jordanienne

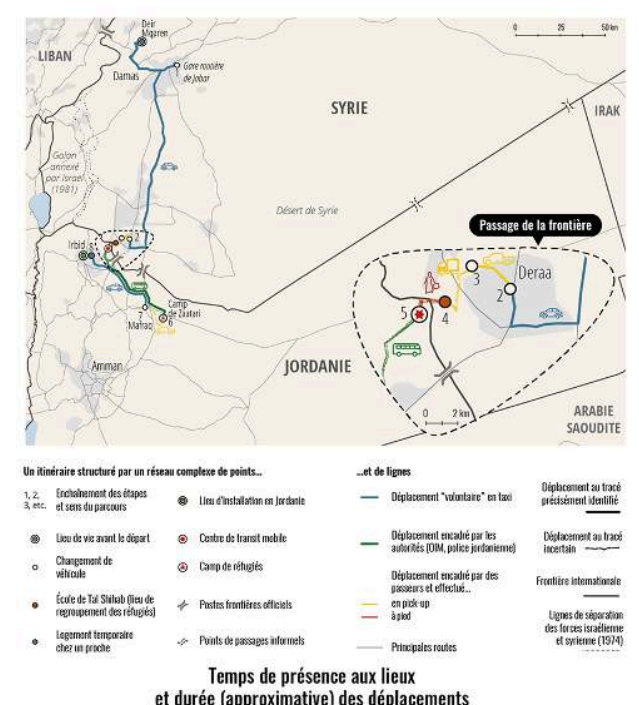

(approximative) des déplacements

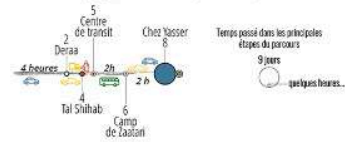

Figure 3. Itinéraire de Yasmin

Itinéraire de Yasmin entre Deir Mqaren et Amman - octobre à décembre 2014 La fermeture des frontières renforce le rôle des passeurs et des autorités dans la structuration des itinéraires
des réfugiés, désormais contraints dentrer par les points de passage informels situés du côté oriental de la

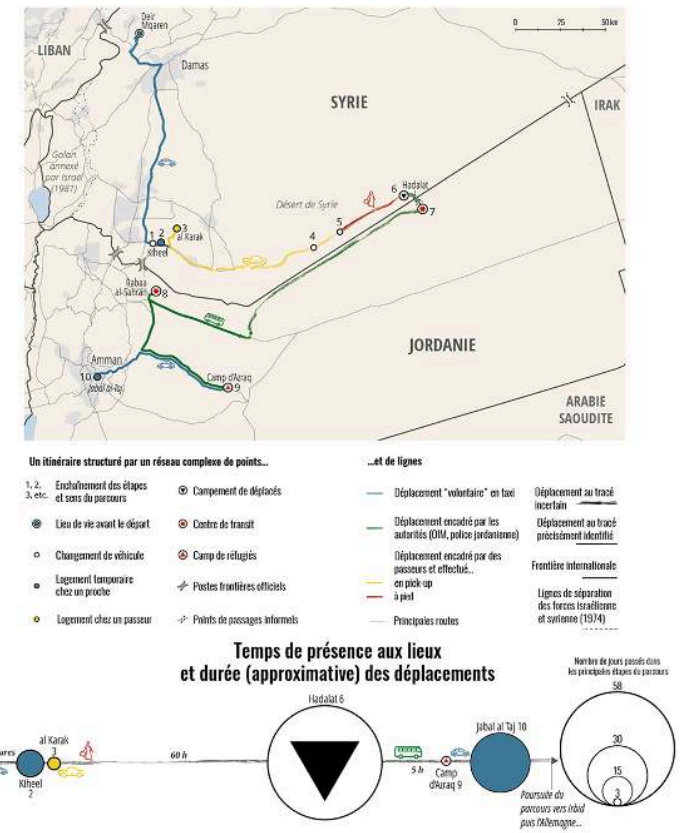


Figure 4. Chaînes relationnelles

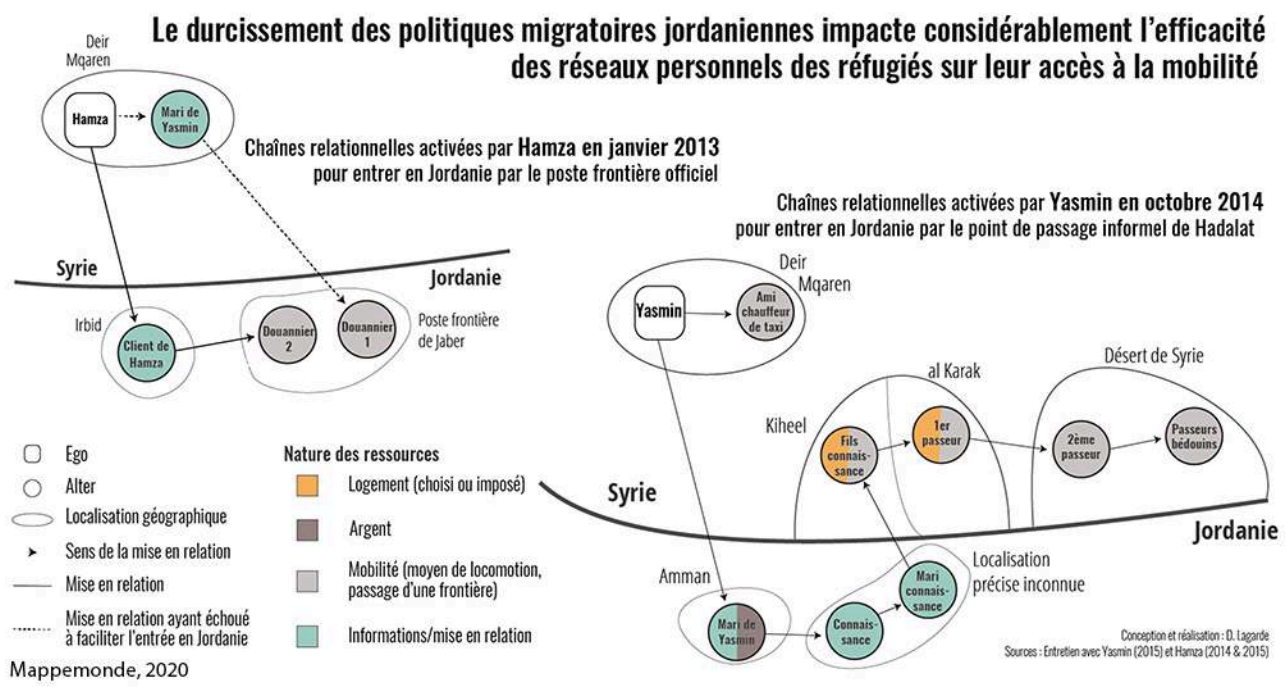

7 En donnant à voir les aspérités qui jalonnent les itinéraires empruntés par les réfugiés, tout en attirant le regard sur des lieux et des acteurs qui, bien qu'étant peu visibles, jouent un rôle clé dans la (re)structuration et le fonctionnement des routes de l'exil syrien, les images géographiques dévoilées ici entendent rendre plus intelligibles des dynamiques migratoires particulièrement complexes et labiles. En plus de révéler les espaces de "friction", les points de blocages et les phases d'arrêt du mouvement engendrées par la fermeture de certains passages, ce travail cartographique vise plus largement à donner du poids aux récits de vie des enquêtés. Dès lors, ces cartes, qui sont à envisager comme des interfaces permettant de "voyager entre lieux et récits " (Caquard et Joliveau, 2016), offrent la possibilité de «matérialiser des parcours de vie fascinants et d'identifier les "points denses" qui les jalonnent » (Caquard et Dimitrovas, 2017).

\section{BIBLIOGRAPHIE}

BACON L., CLOCHARD O., HONORÉ T., LAMBERT N., MEKDJIAN S., REKACEWICZ P. (2016). « Cartographier les mouvements migratoires ». Revue Européenne des Migrations internationales, vol. 32, n 3, p. 185-214. BESSE J.-M., dir. (2010). « Cartographies ». Les carnets du paysage, $\mathrm{n}^{\circ}$ 20, Versailles : Actes Sud. CAQUARD S., JOLIVEAU T. (2016). « Penser et activer les relations entre cartes et récits ». M@ppemonde, $\mathrm{n}^{\circ}$ 118. En ligne :

CAQUARD S., DIMITROVAS S. (2017). « Story Maps \& Co. Un état de l'art de la cartographie des récits sur Internet ». Mappemonde, $\mathrm{n}^{\circ}$ 121. En ligne : https://journals.openedition.org/mappemonde/ 3304

GROSSETTI M. (2011). « Les narrations quantifiées. Une méthode mixte pour étudier des processus sociaux ». Terrains et Travaux. Revue de Sciences sociales, n 19, p. 161-182. 
REKACEWICZ P., TRATNJEK B. (2016). « Cartographier les émotions ». Carnets de géographes, $\mathrm{n}^{\circ} 9$.

TASTEVIN Y.-P., PLIEZ O. (2015). « La discrète filière de l'autorickshaw ». Revue française de socioéconomie, hors-série, $\mathrm{n}^{\circ}$ 2, p. 121-137.

\section{NOTES}

1. Cette bourgade, où vivaient environ 5000 habitants avant le début de l'insurrection en 2011, est située entre Damas et la frontière libanaise. 